\title{
Preconcentrator Module for the Implementation in Optical Gas Measurement Systems
}

\author{
Jürgen Hildenbrand, André Eberhardt, Carolin Peter, Jürgen Wöllenstein \\ Fraunhofer Institute for Physical Measurement \\ Heidenhofstr. 8 \\ D-79110 Freiburg, Germany
}

\author{
Jan Korvink \\ Department of Microsystems Engineering - IMTEK \\ Freiburg Institute for Advanced Studies - FRIAS \\ University of Freiburg Germany \\ Georges- Koehler-Allee 102 \\ D-79110 Freiburg, Germany
}

\begin{abstract}
Fabrication process monitoring in industrial applications, safety and environmental monitoring e.g. for the determination of toxic gases are typical fields of applications in modern gas measurement tasks. In the lower price segment solid state devices like metal oxide based gas sensors are typically used. These sensors are very sensitive but they show high cross-sensitivities and a significant signal drift. Optical techniques distinguish by the compensation of these two disadvantages and it is still possible to get inexpensive absorption spectroscopic systems for e.g. $\mathrm{C}_{2} \mathrm{H}_{4}$. In general these systems are based on thermal IR-sources, a simple absorption cell without optical elements and a detector. The decreasing of the detection limit is mainly reached by increasing of the optical path. We present a new approach to improve the sensitivity of these systems based on a preconcentrating unit mounted to absorption cell.
\end{abstract}

\section{INTRODUCTION}

A rising number of environmental, safety and process applications require measurement systems for gas detection as well as for contamination monitoring of liquids. Examples for such applications are the monitoring of toxic gases and early detection of leakages. Another field is the chemical industry which needs sensor systems for process control. In these applications absorption measurements are an important detection technology which combines high sensitivity, fast response time and high reliability.

In particular infrared spectroscopy facilitates the selective and sensitive measurement of various molecules by their specific absorption. Infrared spectroscopy uses the characteristic absorption of the molecules in the mid infrared and allows the estimation of the species and its concentration. Especially by the absorption at longer wavelengths between 8 and $12 \mu \mathrm{m}$, the so called fingerprint region, the molecules can be measured with highest selectivity. In the last years infrared detection and measurement technologies increases more and more.

Sensitivity enhancement in transmission spectroscopy is typically realised by increasing the optical path I using a optical long path cell according to Beer-Lambert law [1], which states that the optical transmission $\boldsymbol{T}$ is given by

$$
T=e^{-\alpha \cdot c \cdot l}
$$

where $\alpha$ is the absorption coefficient and $\boldsymbol{c}$ the gas concentration. For tasks without the need of a continuous measurement - e.g. one value per hour - a signal enhancement using a cumulating process in a defined period offers the possibility to increase the sensitivity without changing the optical path length.

Preconcentrating itself is a well known method in analytical chemistry and is based on the collection of an analyte during a fixed period. The idea is to enhance the retention time $[2,3]$ of a molecule on a material 
by decreasing its temperature. After a defined cumulating time the retention period have to be reduced by increasing the temperature and the analyte desorbs. The desorbed analyte is typically detected with analytical labour instruments like gas chromatographs.

The basic idea is to combine infrared transmission spectroscopy together with a preconcentrating unit. The preconcentrating unit consists of a chamber for the adsorbent resin and a Peltier-element for cooling and heating.

The principle set-up is shown in figure 1, right. The modulated radiation of a thermal emitter is coupled into a long-path gas cell and is detected by a pyroelectric detector, preamplified and processed by lock-in technique. A specific optical filter must be placed upon the IR detector to select the suitable wavelength for the gas. A filter with a central wavelength of $10.6 \mu \mathrm{m}$ is integrated.

Before the gas molecules enter long path cell they have to pass the preconcentrator unit. The adsorbent resin cumulate with the molecules at a low temperature (figure 1, right, is a snapshot of this state) unless there is a equilibrium between adsorption and desorption. The concentration of the analyte molecules (predominant in an adsorbed state) in the preconcentrator is much higher than the concentration in the gas phase. In the next step the adsorbent resin has to be heated up and the adsorbed molecules will desorb unless the new equilibrium is reached. During the desorption process the gas concentration rises and the molecules flow further to the measurement cell, where the concentration is determined by transmission spectroscopy.

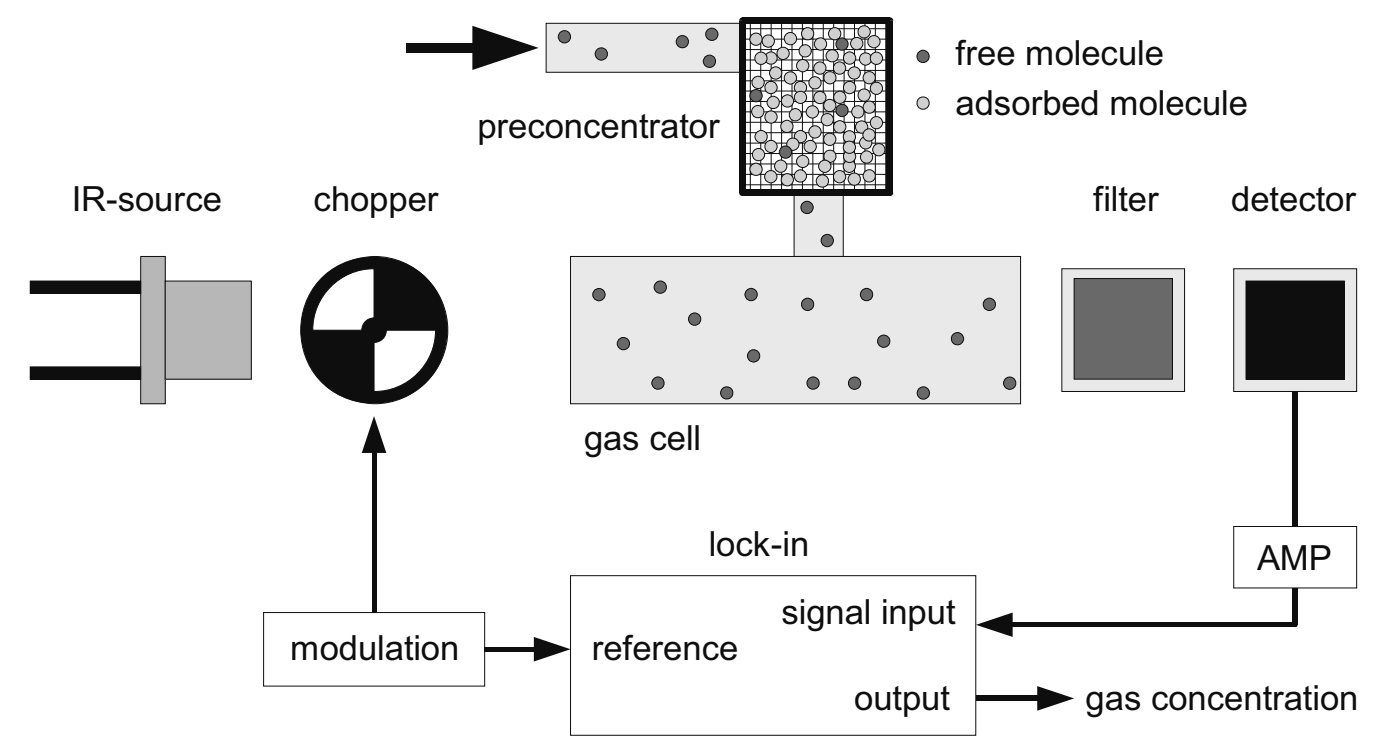

Fig. 1: Schematic set-up of the preconcentrator in combination with a filter photometer. The picture shows the preconcentrator in the accumulation state (High concentration of adsorbed gas molecules). Transmission band of ethylene around a wavelength of $10 \mu \mathrm{m}$. The gas is measured at the central wavelength at $10.6 \mu \mathrm{m}$.

\section{EXPERIMENTAL}

\section{Preliminary Investigations}

A short way between preconcentrator and gas cell is desired in order to ensure that the measurements are performed in a region with a high gas concentration. In larger distances a mixing of the "fresh" desorbed molecules with the low concentration atmosphere in the pipe between preconcentrator and gas cell. In the first tests a separate preconcentrator was used. The module was connected with a small pipe to the gas cell. A peltier element with water cooling was mounted on the preconcentrator unit in order to tune the temperature from $-10^{\circ} \mathrm{C}$ to $\sim 200^{\circ} \mathrm{C}$. The preconcentrator module was equipped with $4 \mathrm{~g}$ of he commercial available adsorbent resin Tenax® TA [4]. 
Using this set-up, an observable desorption peak - and thus a preconcentration process - could be demonstrated. An improved set-up with a minimal distance between preconcentrating and optical gas measurement is required.

\section{Design of the Preconcentrator Module}

The used measurement system is based on a small White-cell with an optical path length of $1.56 \mathrm{~m}$. White-cells are typical used in spectroscopic system to fold the optical path in a small volume [5]. The cell itself is made of aluminium and equipped with gold coated mirrors. The cell has a length of $95 \mathrm{~mm}$, a width of $60 \mathrm{~mm}$ and a height of $36 \mathrm{~mm}$ and is construed for mirrors with a diameter of $22.4 \mathrm{~mm}$ and a radius of curvature of appr. $80 \mathrm{~mm}$. The White-cell frame as well as the top and the bottom cover are made of aluminium (figure 2, left). The gas volume of the cell is $150 \mathrm{~cm}^{-3}$.

The ideal combination of a preconcentrating unit with combined optical transmission spectroscopy is a set-up which has the adsorbent resin as close as possible to the optical path. Therefore a design is intended which replaces the top cover of the White cell and desorp the molecules from the top side into the cell. Figure 2 (right) shows the lower part of the preconcentrator. The bottom of this part is mounted on the White-cell. The chamber for the adsorbing resin is positioned in the middle part. Small pins are placed in this chamber in order to increase the thermal coupling from the metal body to the resin.
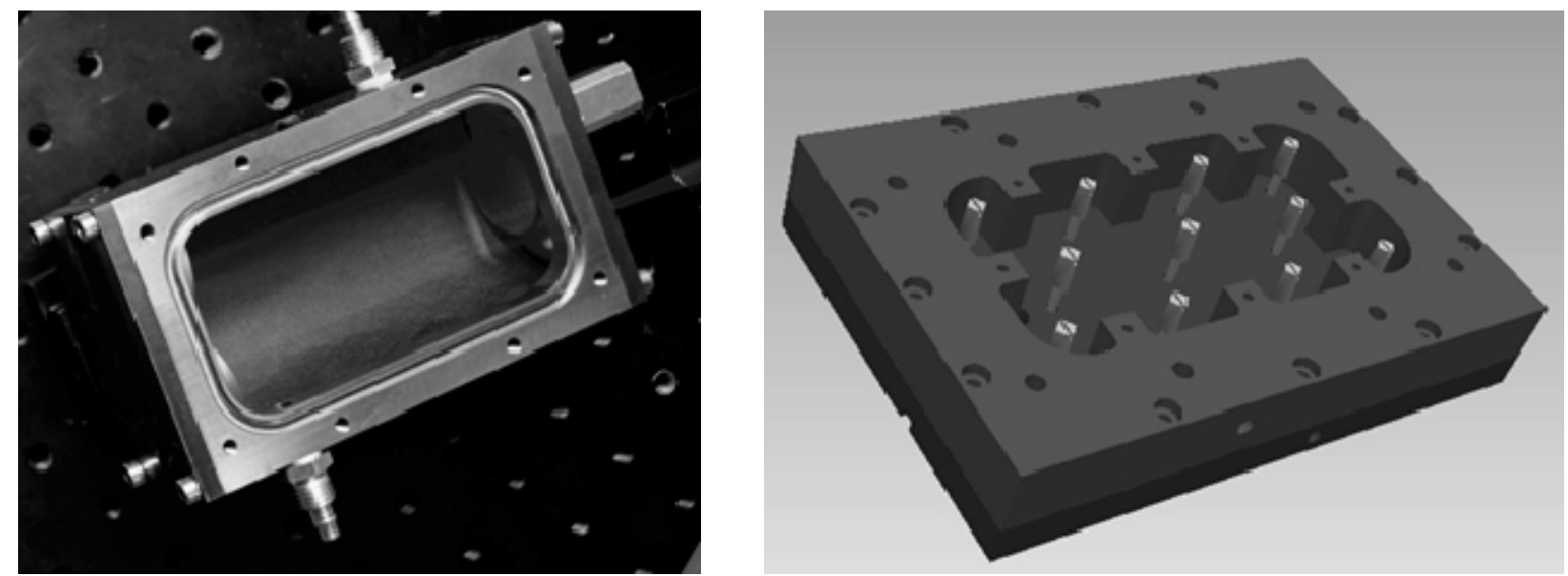

Fig. 2: (left) White-cell with $1.6 \mathrm{~m}$ optical path length. (right) Resin chamber of the preconcentrator modul. On the top of this chamber a Peltier-element with water cooling is integrated. The thermal distribution during the heating and cooling process is investigated using FEM (figure 3).

The preconcentrating module should reach a minimal temperature of $-18.9^{\circ} \mathrm{C}$ and a maximal temperature of $150^{\circ} \mathrm{C}$. The estimation of the power consumption and the thermal behaviour were investigated using the FEM software COMSOL. Figure 3 (a) shows the main model consisting the White-cell body and the preconcentrator unit. The preconcentrator unit is directly attached instead of the top cover to the Whitecell. The White-cell body is mounted on four spacers (combination steel/ceramic). A constant temperature of $22^{\circ} \mathrm{C}$ is applied at the end of theses spacers. The thermal power is homogenously applied at the interface between Peltier-element and the preconcentrator module. The Peltier-element itself is not considered in the simulation. Further the heat-flux to the ambient is estimated with a heat transfer coefficient of $5 \mathrm{~W} /\left(\mathrm{m}^{2} \mathrm{~K}\right)$. The implementation of the bulk material properties is noncritical. The uncertain values are the ones for the adsorbing resin. Especially the thermal conductivity, which is a significant value for the thermal coupling, of such powder is unknown. Alternatively a value of $22^{\circ} \mathrm{C}$ is used to approximate a "typical" thermal isolating material. The simulation showed that a power in the order of $15 \mathrm{~W}$ is required for the minimal temperature of $-18.9^{\circ} \mathrm{C}$ and a power of $150 \mathrm{~W}$ for the maximal temperature of $152^{\circ} \mathrm{C}$. The resin chamber shows a uniformly temperature distribution in a steady state (figure 3). 


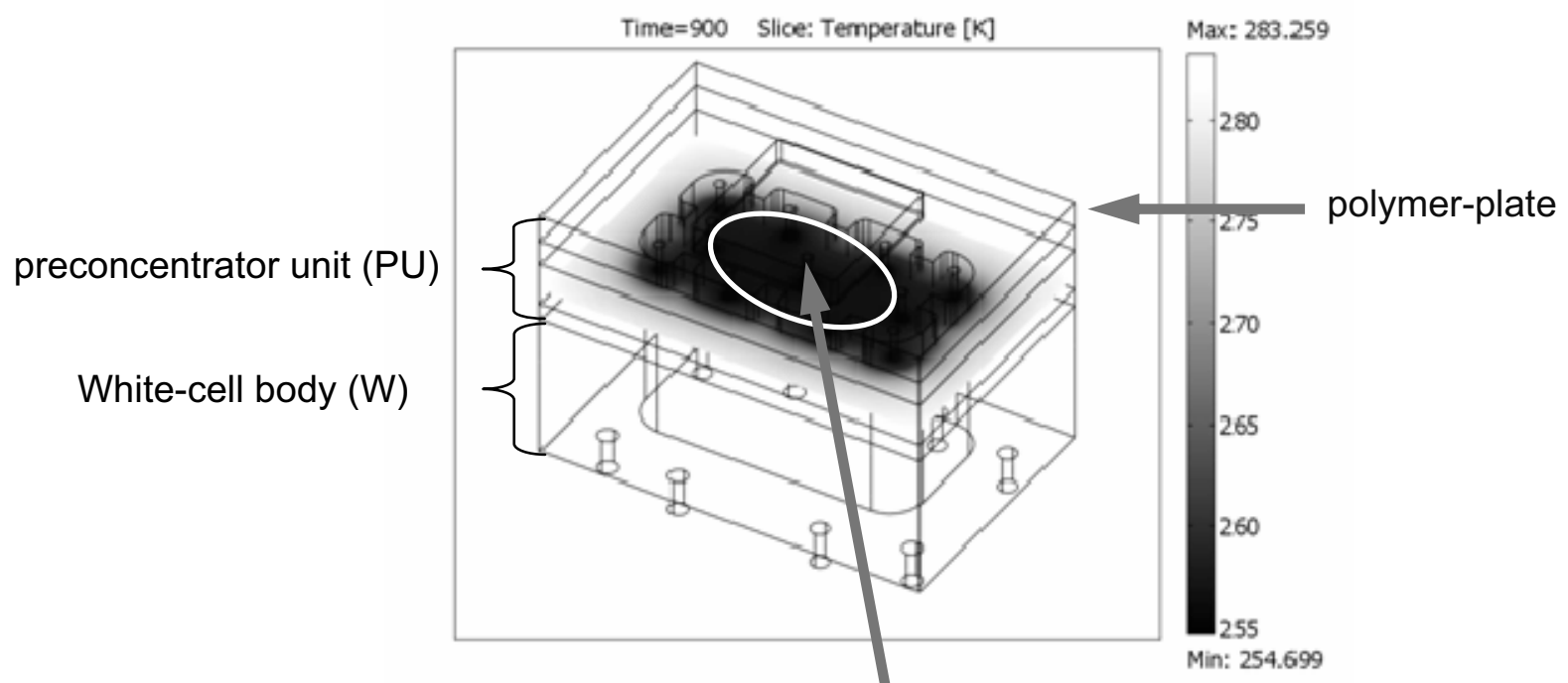

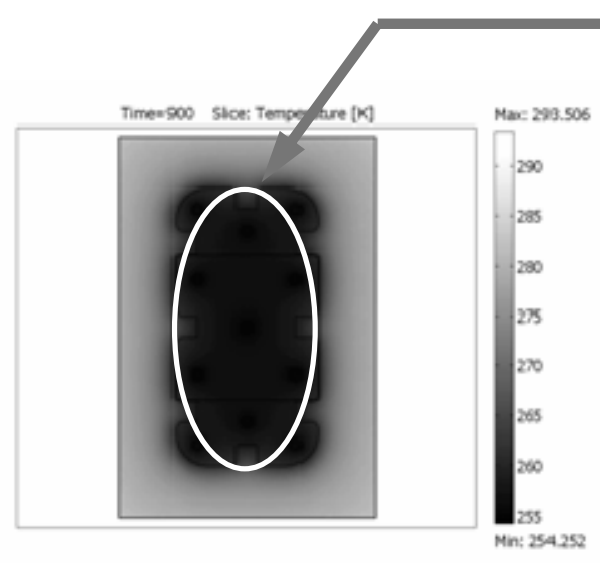

(b) (a)

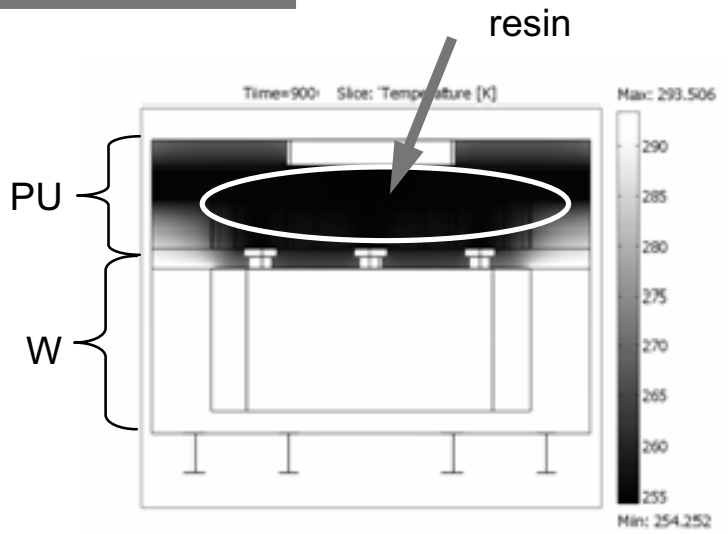

(c)

Fig. 3: (a) FEM-model of the White-cell / preconcentrator unit. Temperature distribution at the end of the cooling sequence at the center of the resin chamber. (b) Top view of the of the temperature distribution of the same region during the cooling process. (c) Vertical cross-section through the White-Cell and the preconcentrator.

\section{Measurement Set-Up}

Figure 4 show the complete measurement set-up with the preconcentrator module mounted on the Whitecell. A thermal emitter from lon-Optics which is mounted in a sealed housing is used. Between the emitter and the White-cell a mechanical chopper is positioned. The IR-source was modulated with the chopper at a frequency of $10 \mathrm{~Hz}$. A pyroelectric detector with a sensitive area of $3 \times 3 \mathrm{~mm}^{2}$ from Infratec with an integrated preamplifier was used for the detection of the optical power. The preamplified detector signal was measured using a data-acquisition card (NI USB-6212) and evaluated with a software Fourier-filter at $10 \mathrm{~Hz}$. The gas concentrations were realised using a gas mixing station equipped with pure $\mathrm{N}_{2}$ and 1000ppm ethylene in $\mathrm{N}_{2}$. The gas flow was controlled to $0.2 \mathrm{l} / \mathrm{min}$. Besides the Fourier-filter a software lock-in-amplifier based on the data acquisition card as well as a commercial hardware lock-In-amplifier (7265DSP from Signal Recovery) were tested for this application. The need of the lock-in technique depends here strongly on the constant velocity of the chopper. If the influence of the variation in the chopper frequency dominates in the detector signal, a lock-in technique is essential in order to achieve a sufficient signal to noise ratio.

If the chopper creates a stable frequency the single frequency-Fourier-filter will be preferred for signal analysis. For this task some test measurements with a waveform generator at a frequency of $10 \mathrm{~Hz}$ and a signal amplitude of $5 \mathrm{mV}$ (similar to the detector signal) were carried out. For this signal the Fourier-filter based method has a signal to noise ratio (SNR) of $3.77^{*} 10^{-6}$ with an integration time of 4 seconds. In 
comparison to the Fourier-filter implementation the hardware-lock-in-amplifier has a SNR of $5.38^{*} 10^{5}$, which is almost an order of magnitude lower. Comparison measurements with the system - including the mechanical chopper - showed a similar characteristic $\left(\mathrm{SNR}_{\text {Fourier }} \sim 2.0^{*} 10^{5}\right.$ and $\left.\mathrm{SNR}_{\text {lock-in }} \sim 1.8^{*} 10^{5}\right)$. Motivated by the more efficient implementation the Fourier-based method was used in the final system.

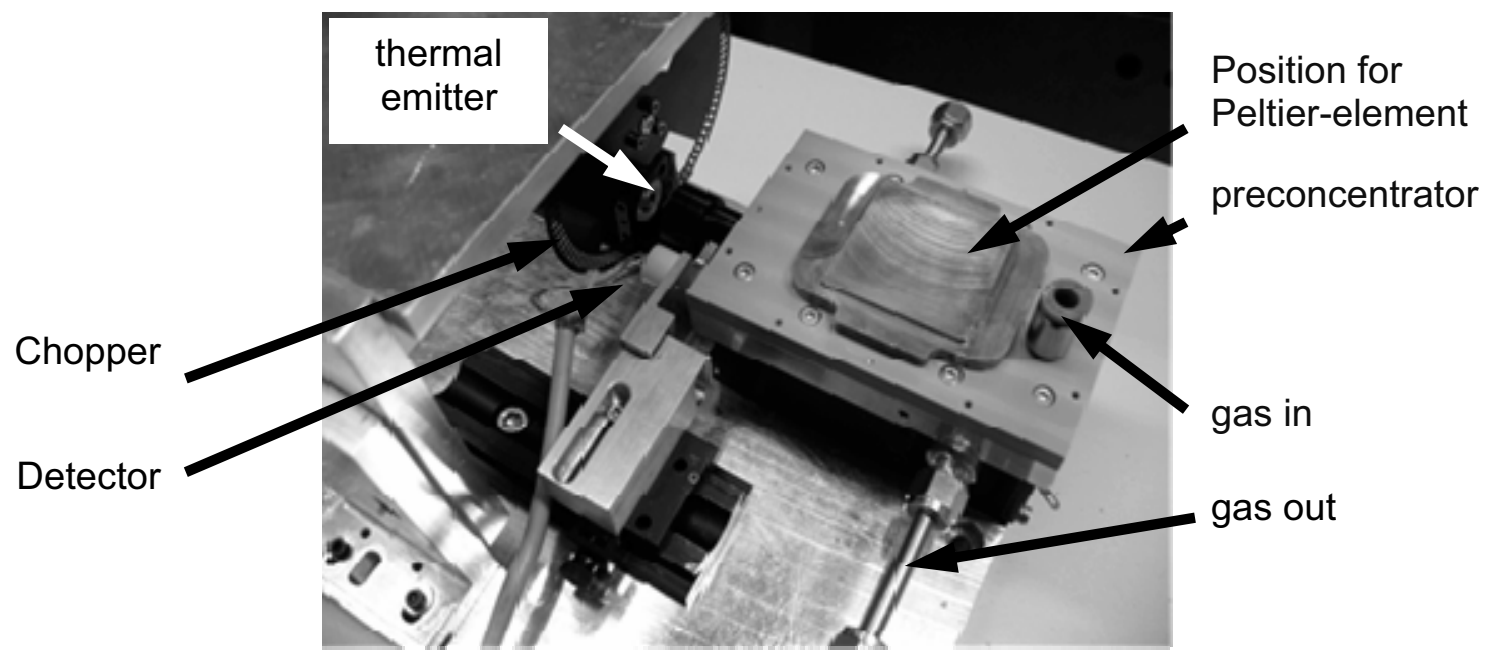

Fig. 4: Measurement set-up for the preconcentrator investigations. The Peltier-element is removed in this photo in order to have a better overview of the set-up.

\section{Measurements}

First, a investigation concnerning the system performance without preconcentrating were performed. A concetration profile from $0 \mathrm{ppm}$ to $50 \mathrm{ppm}$ in $10 \mathrm{ppm}$ and back were applied. The mean value was taken during a period of $40 \mathrm{~s}$. The measurement is shown in figure 5 . With these parameters a detection limit of $2 \mathrm{ppm}$ was realised. The present set-up is not drift compensated. The drift is less than $1 \mathrm{ppm} /$ hour. For a "real" system application a reference detector and a temperature correction has to be implemented.

In the next step the measurements with the preconcentrator were performed. A constant gas flow with a concentration $400 \mathrm{ppm}$ was used in order to ensure a high fractional coverage of ethylene on the adsorbent resin. That was necessary due to the bad thermal coupling between the adsorbent resin and the heat exchanger. The average thermal conductivity of the resin - and thus the thermal coupling to the heat exchanger - was much higher assumed in the simulation than it is. So, only a small part of the adsorbent resin could be cooled and heated fast enough to indicate adsorption and desorption processes.
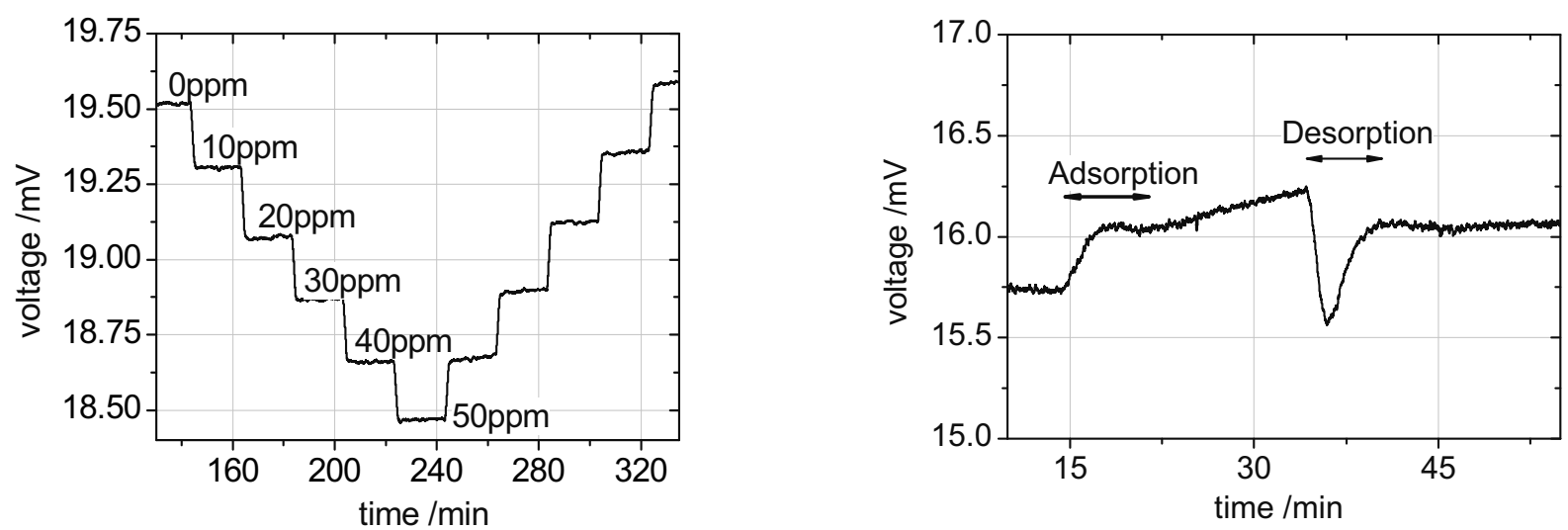

Fig. 5: (left) Etyhlene measurement at different concentrations. The detection limit is $2 \mathrm{ppm}$. (right) Ethylene measurement using the preconcentrator Module. In order to ensure a high ethylene coverage on the adsorbent resin in "short time", a gas concentration of $400 \mathrm{ppm}$ was chosen. The desorption concentration is $\mathbf{4 7 4 . 9} \mathrm{ppm}$. 
Some estimations, based on the retention time in dependence of the temperature yield in a improvement factor of about 3.9 in comparison to the normal concentration. In the measurements an improvement factor of 1.19 could be reached. So, only about $30 \%$ of the adsorbent resin is well coupled to the heat exchanger. The improvement of the thermal coupling will increase the preconcentrating process significantly.

\section{SUMMARY AND OUTLOOK}

A novel combination of a filter photometer with a preconcentrating unit was presented. A detection limit of $2 \mathrm{ppm}$ ethylene was achieved with the basic system (without preconcentrator) The preconcentrating effect was shown with ethylene using a gas concentration of $400 \mathrm{ppm}$ in order to reach a high fractional coverage in a short time. The resulted desorption concentration is $474.9 \mathrm{ppm}$. Nevertheless, the amount of the implemented adsorbent resin should yield in a significant higher desorption signal. It is assumed that the thermal coupling from the inner part of the resin to the temperature controlling element is not high enough. So, only a part of the resin has the intended temperature change and thus only this part is able to accumulate and to desorb the ethylene molecules. In the next steps the thermal coupling of the adsorbent resin to the heat exchanger will be investigated in detail using FEM-simulations. With the optimised redesign and thus the thermal coupling of the complete resin to the heat exchanger an improvement factor of 4 is expected.

\section{ACKNOWLEDGEMENTS}

This work was partly funded by the BMBF and the Fraunhofer Gesellschaft.

\section{REFERENCES}

[1] Peter W. Atkins, Physikalische Chemie, Wiley VCH (2001)

[2] John J. Manura, Calculation and Use of Breakthrough Volume Data, SIS,

http://www.sisweb.com/index/referenc/resin10.htm (2009)

[3] Anonín Kroupa, J. Dewulf, H. V. Langenhove, I. Víden, Breakthrough characteristics of volatile organic compounds in the -10 to $+170 \circ \mathrm{C}$ temperature range on Tenax TA, determined by microtrap technology, Journal of Chromatography A, 1038(2004)

[4] SIS Inc., Tenay TA Adsorbent Resin Physical Properties, http://www.sisweb.com/index/referenc/tenaxta.htm (2009)

[5] J.U. White, Long Optical Paths of Large Aperture, J. Opt. Soc. Am. 32:285 (1942) 